\title{
Paget-von Schroetter-Syndrom als Arbeitsunfall
}

A. Böcher*, M. Müller*, P.Scheffler** L. Zell ${ }^{\star * \star}$, A. Buchter*

*Institut und Poliklinik für Arbeitsmedizin, Universität des Saarlandes, Homburg/Saar (Leiter Prof. Dr. med. A. Buchter),

${ }^{* *}$ Innere Medizin III, Abt. Angiologie, Universitätsklinikum des Saarlandes, Homburg/Saar

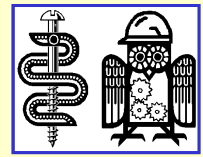

${ }^{* * \star}$ EnBW Service GmbH, Arbeitsmedizinischer Dienst, Karlsruhe

Einleitung: Das Paget-von Schroetter-Syndrom ist eine tiefe Venenthrombose der Vena axillaris und/oder der Vena subclavia. Sie ist verbunden mit den typischen Zeichen einer tiefen Venenthrombose, wie Schmerz, Schwellung, Zyanose und evtl. erkennbare Kollateralvenenzeichnung. Neben iatrogenen Ursachen, Malignomen, Hyperkoagulabilität und anatomischer Prädisposition kommt eine Auslösung durch ruckartige Krafteinwirkung in Frage. Hat die Belastungseinwirkung nachweislich während einer versicherten Tätigkeit stattgefunden, ist diese Erkrankung beim zuständigen gesetzlichen Unfallversicherungsträger als Arbeitsunfall zu melden. (1-3)

Anamnese: Ein 35-Jähriger Maler und Lackierer verspürte beim Gerüstabbauen einen reißenden Schmerz in der rechten Schulter, nachdem er ein Brett über Kopf mit einer ruckartigen Bewegung aus der Verankerung herausstemmte. Am Folgetag bestand eine Schwellung und Schmerz im gesamten rechten Arm.

Befunde: Klinische Untersuchung: schmerzhaften Schwellung und vermehrte Venenzeichnung re Schulter. Phlebographie: Abbruch der Kontrastmittelsäule Übergang V.axillaris/V. subclavia re, fehlende Darstellung der V. subclavia im gesamten Verlauf. CT des oberen Thorax: Ausschluss venöse Abflussstörung. Labor: Ausschluss Resistenz gegen aktiviertes Protein C (APC-Resistenz) und Prothrombin 20210A-Mutation. Farbcodierte Duplexsonographie (Abb 1): Rekanalisation des Gefäßes mit verbleibender Randthrombose, Wandverdichtung und typische Verdickung ventral lateral und Intimaeinriss (ca. 5 Wochen nach Trauma). Diagnose: Paget-von Schroetter-Syndrom rechts bei Intimaeinriss der rechten Vena subclavia infolge eines Arbeitsunfalles.
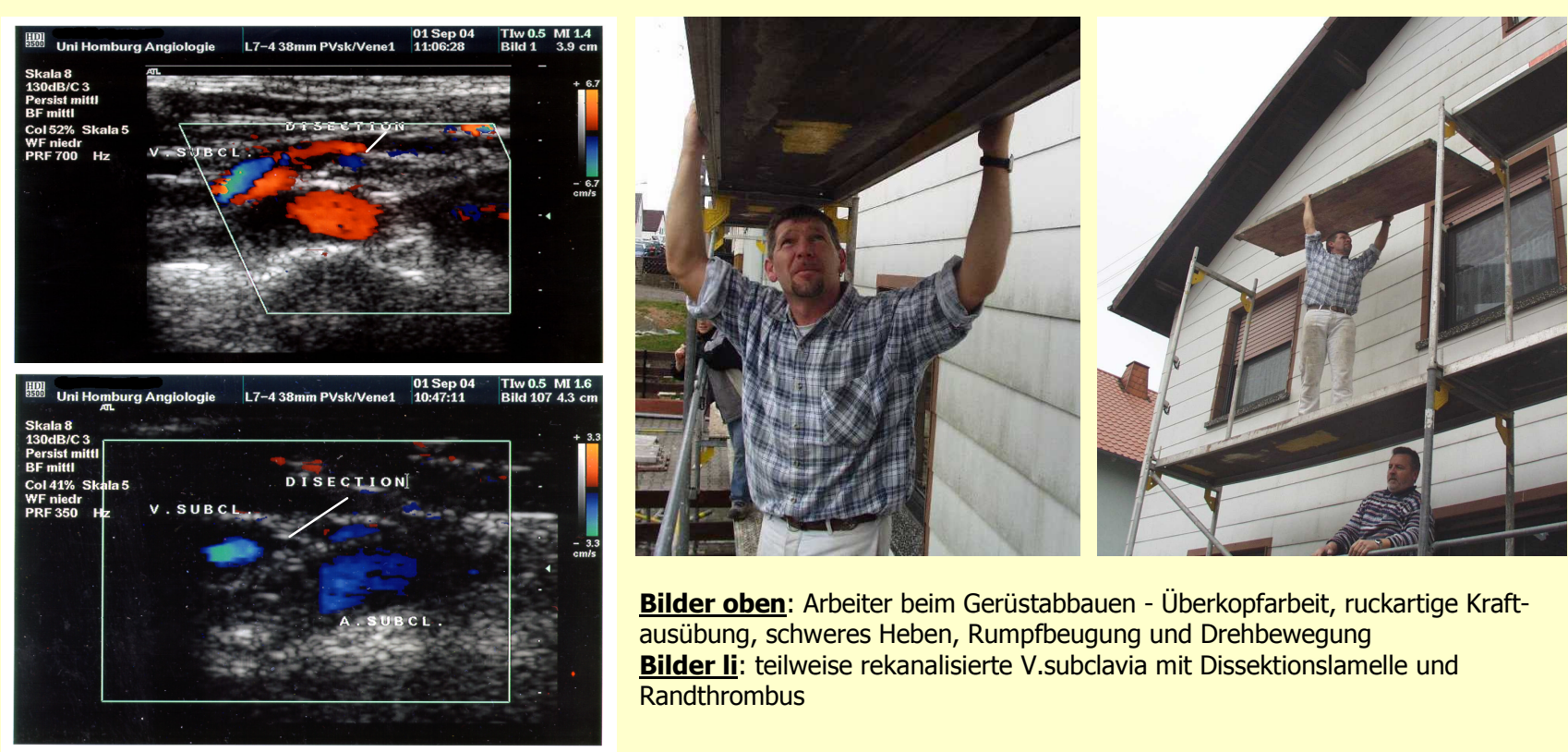

Bilder oben: Arbeiter beim Gerüstabbauen - Überkopfarbeit, ruckartige Kraftausübung, schweres Heben, Rumpfbeugung und Drehbewegung

Bilder li: teilweise rekanalisierte V.subclavia mit Dissektionslamelle und Randthrombus

Therapie und Verlauf: Kompressionsverband. Orale Antikoagulation über 3 Monate. Rasche Rekanalisierung der V.subclavia Nach Beendigung der Antikoagulation sollte eine ergänzende hämostaseologische Diagnostik erfolgen (ATIII, Protein C und S).

Diskussion: Traumatische Venenläsionen durch Unfälle, körperliche Überanstrengung oder sportliche Betätigung mit der Folge eines Paget-von Schroetter-Syndromes (auch „Thrombose par effort" genannt) sind eher selten. Durch eine gezielte Anamnese war hier ein unfallartiges Ereignis während der Arbeit zu eruieren. Mittels Duplexsonographie konnte zudem eine Intimaläsion an typischer Stelle als Folge einer traumatischen Einwirkung beschrieben werden. Weitere Ursachen konnten durch eine CT und Gerinnungsdiagnostik weitgehend ausgeschlossen werden. Zell et al. (2) haben in früheren Arbeiten erstmals durch eine lückenlose Beweiskette Thrombosen der Vena subclavia als Arbeitsunfall zur Anerkennung bringen können. In Fällen ohne akutes Ereignis aber Bezug zur Arbeitsanamnese (wiederholte und außergewöhnliche Kraftanstrengung über mehrere Arbeitsschichten) wurde die Erkrankung als BK nach §9, Abs.2, SGB VII angezeigt („Öffnungsklausel"). Eine Anerkennung als BK ist bisher mit der Begründung der fehlenden Gruppentypik nicht erfolgt. Nach mehrtätiger (subklinischer) Vorschädigung ist der Tag mit Eintreten der Intimaschädigung und Thrombose als Tag des Arbeitsunfalles anzusehen und anzuzeigen.

Fazit: Bei unklarem Auftreten eines Paget-von Schroetter-Syndroms, insbesondere bei im Berufsleben stehenden jüngeren Menschen, muss immer an eine berufliche Verursachung gedacht werden. Diese ist nachweisbar durch eine gezielte Arbeitsanamnese mit Eruierung eines traumatischen Ereignisses sowie einer duplexsonographisch nachweisbaren Intimaverletzung. Die Erkrankung ist als Arbeitsunfall bei der zuständigen Berufsgenossenschaft anzuzeigen.

Literatur

(1) ZELL L, KINDERMANN W, MARSCHALL F, SCHEFFLER P, GROSS J, BUCHTER A. Paget-Schroetter syndrome in sports activities. Case study and literature review. Angiology 2001; 52: 337-342.

(2) ZELL L, SCHEFFLER P, HEGER M, BUCHTER A. Paget-von Schroeter-Syndrom als Arbeitsunfall. Dtsch Med Wschr 2001: 126: $326-328$.

(3) ZELL L, SOMMERFELD A, BUCHTER A. Das Paget-von Schroetter-Syndrom. Zum 100. Todesjahr von Sir James Paget und zur 50-jährigen Namensgebung des Syndroms.

Dtsch Med Wschr 1999: 124: 948-951. 